\title{
H2O Metacomputing - Jini Lookup and Discovery
}

\author{
Dirk Gorissen, Gunther Stuer, Kurt Vanmechelen, and Jan Broeckhove \\ Antwerp University, Middelheimlaan 1, 2020 Antwerp, Belgium \\ Dirk.Gorissenastudent.ua.ac.be, \\ \{Gunther.Stuer, Kurt.Vanmechelen, Jan.Broeckhove\} @ua.ac.be
}

\begin{abstract}
Because of its inter-organisational, collaborative use of computational resources, grid computing presents a severe interoperability challenge to grid application developers. Different middleware technologies need to be bridged in order to fully utilise the power the grid provides. This paper describes a bridge between two such middlewares: The $\mathrm{H} 2 \mathrm{O}$ Metacomputing Framework and Jini technology. The paper details how $\mathrm{H} 2 \mathrm{O}$ resources may be registered, discovered and used as Jin 1 services. Both technologies are introduced, design decisions discussed and a fully functional implementation presented.
\end{abstract}

\section{Introduction}

The term Grid refers to an infrastructure that enables the integrated, collaborative use of (high-end) computers, networks, databases, and scientific instruments owned and managed by multiple organisations [1]. Consequently, by definition, grid computing implies collaboration and coordination across organisational and geographical boundaries. This increased scale in turn means that the environment we are dealing with is increasingly heterogenous and that interoperability becomes a necessity rather than a feature. This interoperability manifests itself at three levels: the underlying network architecture, the middleware, and the administrative and organisational structure.

This paper will concentrate on the middleware. It will describe a bridge between two grid technologies: Emory University's H2O Metacomputing Framework [2] and Sun Microsystems' Jini technology [3]. More concretely we will describe a way to automatically publish $\mathrm{H} 2 \mathrm{O}$ resources as Jini services in order to extend the reach and flexibility of the resulting middleware.

We start off with an introduction to both $\mathrm{H} 2 \mathrm{O}$ and Jini in sections (2) and (3) followed by a brief discussion of their integration in section (4). Section (5) then goes on to analyse the different design decisions that need to be made while section (6) describes the actual implementation itself. We conclude the paper in section (7) with a summary and an outline of future work.

\section{$2 \mathbf{H 2 O}$}

$\mathrm{H} 2 \mathrm{O}$ is developed by Emory University and is a novel component-based, serviceoriented framework for distributed metacomputing. Adopting a provider-centric view

\footnotetext{
${ }^{1}$ Jini and Java are registered trademarks of Sun Microsystems.
} 
of resource sharing it provides a lightweight, general-purpose, Java based, configurable platform. $\mathrm{H} 2 \mathrm{O}$ adopts the microkernel design philosophy: Resource owners host a software backplane (called a kernel) onto which owners, clients, or third-party resellers may load components (called pluglets) or component-suites that deliver value added services without compromising owner security or control [2]. For easy identification every $\mathrm{H} 2 \mathrm{O}$ resource (kernel or pluglet) is identified by a unique URL (eg: http://myKernel:7799/).

The relationship between a kernel and its pluglets is best understood in terms of an analogy with servlets. Servlets are Java entities used to perform cgi-like processing tasks and need to be deployed within a servlet-container (eg: Tomcat from the Apache Jakarta project). In the same way as Tomcat is a container for servlets, $\mathrm{H} 2 \mathrm{O}$ is a container for services, aka pluglets.

$\mathrm{H} 2 \mathrm{O}$ was designed to cater for lightweight, general purpose, loosely coupled networks where resources are shared on a P2P basis. Consecutively, H2O does not target large scale computer networks (like Globe [4] does) nor does it aim for adoption in high speed, performance critical networks (like TAO [5]).

While the kernel is completely under the resource owner's control, authorised clients may configure, securely use and even freely deploy new pluglets into the kernel. It is this last aspect that makes $\mathrm{H} 2 \mathrm{O}$ unique. Namely, by separating the service provider from the resource owner it becomes possible for any authorised third party to deploy new services in a running kernel.

\section{Jini}

Jini was developed by Sun Microsystems in the late 1990's and many of the people who created and championed it are the same people who originally created and championed certain aspects of Java. Jini is a distributed, service-oriented system based on the idea of federating groups of users and the resources required by those users. Jini's overall goal is to turn the network into a flexible, easily administered tool on which resources can be discovered and used by human and computational clients.

Jini services are represented as Java interfaces and are registered with the Jini Lookup Service (LUS). The LUS plays the role of the service broker and is the bootstrapper of a Jini federation. It is automatically discovered through the discovery protocol (spontaneous computing) and its services are queried through the lookup protocol. Jini further supplies a distributed transaction and event manager, pluggable transports, an extensive (mobile code) security framework and a self-healing mechanism based on leasing. The latter means that each service is granted a lease when it joins the Jini federation and it is up to the service to renew this lease as long as it wants to remain part of the federation. In this way zombie services are automatically removed. All this makes Jini a very robust platform for grid computing, one that safely accommodates the Eight Fallacies of Distributed Computing [6].

A big advantage of Jini is its flexibility. Jini is basically a set of APIs that define how services connect to one another and not what those services are, what they do or how they work. Applications of Jini technology range from autonomous robotic swarms [7], grid middleware (JGrid [8, 9], ICENI [10], JISGA [11]), HPC [12], to online air ticket reservation systems (Orbitz.com). 
Jini also enjoys a large community with numerous projects that extend the reach and capabilities of the Jini framework. Examples include Judy [13] (integration of Jini and Webservices) and Rio [14] (provides dynamic service containers, monitoring, load distribution, QoS enforcement and Resource Cost analysis mechanisms).

\section{Building Bridges}

The features and properties of Jini such as spontaneous, ad hoc networking, failure resilience, service discovery and the service-oriented programming model make it a very suitable base for creating dynamic, reliable grid systems. Despite this fact, little work has been done in creating Jini-based grid systems. This is mainly due to a number of persistent misconceptions, ie. Jini is just for devices, it is Java only, it wont work with webservices, it is not secure, Jini is tied to RMI, etc. All of which have been proven to be wrong [15].

While both $\mathrm{H} 2 \mathrm{O}$ and Jini provide service-oriented resource abstraction layers, the major difference between the two is the conceptual approach. $\mathrm{H} 2 \mathrm{O}$ strives to group services into one, homogeneous container. This provides a uniform, well defined paradigm for services to operate in, making tasks such as authentication and resource access easier to manage. In contrast, Jini is much more distributed and loosely coupled in nature, placing no restrictions on how services are deployed. Another difference is that, in addition to the resource abstraction, Jini provides discovery and registration protocols to enable dynamic, spontaneous computing.

It should now become clear that both technologies perfectly complement each other. Jini excels in exactly that what $\mathrm{H} 2 \mathrm{O}$ lacks (a registration and discovery scheme) and vica versa. We will now go on to describe how $\mathrm{H} 2 \mathrm{O}$ can be extended with Jini registration and discovery support. In this way a prospective $\mathrm{H} 2 \mathrm{O}$ user no longer needs a priori knowledge of the location of $\mathrm{H} 2 \mathrm{O}$ resources (kernels and pluglets).

\section{Design Decisions}

Before we outline how we implemented such registration support, a discussion concerning the different design decisions is in order.

Our investigation will be subdivided into four sections: the 'What', the 'Who', the 'When' and the 'How'.

\subsection{What to Register}

The first question we need to ask is what do we want to register. Simply the kernel, or the individual pluglets as well? The second choice results in each pluglet receiving a separate entry in the LUS that must be kept up to date. The complications that arise from this are discussed later on. The other option would be to only register the kernel and keep the pluglets safely hidden. A discovery wrapper class could then be used to hide kernel access methods, effectively making pluglet discovery transparent for the client. However, this mechanism does introduce significant overhead and restricts the 
discovery of $\mathrm{H} 2 \mathrm{O}$ services to clients with $\mathrm{H} 2 \mathrm{O}$ access only (the wrapper will require $\mathrm{H} 2 \mathrm{O}$ login credentials). We therefore opt for the first method.

\subsection{Who Will Do the Registering}

Next we have to ask ourselves who will ultimately bear the registration burden. This is important since different entities have access to different information and thus have different registration powers. We will consider 3 candidates: The kernel itself, a pluglet, or any interested third party.

The first is not desirable since it requires altering the kernel codebase and would break the $\mathrm{H} 2 \mathrm{O}$ microkernel philosophy. A kernel and its pluglets should be unaware of any registration scheme.

Another possibility pushes the registration responsibilities completely away from $\mathrm{H} 2 \mathrm{O}$, towards any interested third party with enough information to do the job. For example: any Jini knowledgeable entity who knows the kernel or pluglet URL is free to register this information in the Jini lookup service. A client with login access to $\mathrm{H} 2 \mathrm{O}$ could do even more: it can obtain a kernel/pluglet reference and register this together with the URL. However, registering a live kernel reference would allow unrestricted access to the $\mathrm{H} 2 \mathrm{O}$ kernel, something that should be avoided. This options raises some leasing questions as well. Namely, how will the third party guarantee that the proxy it registers actually represents a live kernel?

The third option can be seen as a mixture of the above two. The idea is to let a designated registration pluglet do the registering. This way we don't have to litter the kernel with registration code and still maintain some degree of control. The lease problem is also solved though it involves a little more work. Concentrating all registration services in one designated pluglet means that this one pluglet is then responsible for handling all Jini leases. This implies that:

1. The registration pluglet needs to keep track of pluglet state changes and all the leases it holds so far. When a pluglet is destroyed the registration pluglet needs to cancel the relevant lease.

2. If the registration pluglet dies or is destroyed for some reason all the other pluglets are automatically removed from the LUS since their leases are no longer renewed.

(1) is possible through some extra coding and we we assume (2) to be unlikely.

\subsection{When to Register}

A third, overlapping question is "When should $\mathrm{H} 2 \mathrm{O}$ resources be registered?". To answer this we have to discriminate between kernels and pluglets. We commence with the kernel case, kernel registration can occur on startup or at run time.

The option that comes to mind first is to register the kernel automatically on startup. The only sensible way to achieve this is through a registration pluglet that is automatically deployed during the kernel bootstrap process. The pluglet then performs the registration as soon as it gets loaded during kernel startup.

The other option is to register at runtime. Again a registration pluglet is used but the kernel now has to be registered through a manual call to a method like regKernel ( ) . 
Registering pluglets is handled similarly. Pluglets can be registered at deployment time or at some later stage in their life. A registration pluglet can take care of both cases by (1) listening for kernel generated pluglet deployment events and performing the registration when they occur or (2) by providing a method like registerPluglet (String name) that can be manually invoked.

\subsection{How to Register}

Having decided who will do the registration and at what time, we can now consider how the actual registration will take place. We list the different possibilities.

Resource URL. The easiest option is to simply use resource URLs. Each H2O kernel and pluglet is identified by a unique URL (eg: http://myhost:7799/myPluglet). Registering these means that the client no longer needs a H2O URL on startup, it can discover one itself. A wrapper class can be written that discovers a LUS, finds the appropriate $\mathrm{H} 2 \mathrm{O}$ service (kernel or pluglet) and uses the thereby obtained URL's to perform a $\mathrm{H} 2 \mathrm{O}$ login.

Though easy to implement, this approach does have a number of downsides:

- A bundle of URLs contain no information about the underlying kernel. Although extra meta data can be attached in the form of Jini Entry objects this metadata is constrained to being semi-static in nature (eg: version, name, application domain, underlying platform, etc). More interesting, faster changing attributes such as load, available pluglets, etc would require an additional login step first.

- It is impossible to perform service interface based lookups, the standard way of discovering services in a Jini system.

Raw Remote Reference. Completely orthogonal to registering a URL is registering a live, Java, remote reference which points back to a kernel/pluglet. The obvious objection here, among others, is that distributing such a reference gives anybody, free, unconditional access to the kernel. Effectively bypassing every $\mathrm{H} 2 \mathrm{O}$ security mechanism.

Resource URL \& Service Interfaces. This option intends to be a compromise by ensuring that the registered proxy provides the resource URL and 'implements' every service interface. The catch is that the proxy only provides the interface, but not its implementation (invocations would throw exceptions). This 'empty box' approach allows interface based lookup while forbidding unrestricted access.

Resource URL \& Dynamic Information. A downside of the URL approach is that the resulting proxy tells us nothing about the kernel it represents. It would be nice if a client could choose a particular kernel based on dynamic attributes such as load, number of pluglets, etc. Enabling this requires registering the kernel under an interface that, besides the kernel URL, provides methods to retrieve such dynamic information. The 
implementation of these methods would be through a remote stub that, in accordance with the microkernel design philosophy, calls back to a pluglet that provides dynamic kernel monitoring. In this case the proxy that is registered is called a smart proxy since it is more than just a simple stub. Note that this option assumes such dynamic information is allowed to be freely accessed. This option can also be combined with the previous one.

Login Enabled Smart Proxy. A final option is to extend the smart proxy above with a method that logs into the kernel the proxied resource belongs to without requiring an explicit resource URL, as is usually the case. In practice this means that, in the kernel case, the client would first lookup a proxy representing a kernel and then use it to log into the $\mathrm{H} 2 \mathrm{O}$ system by calling getKernel Context (Credentials). An explicit URL parameter is no longer needed since the proxy contains one.

In the pluglet case the proxy additionally implements all functional interfaces (thus allowing service interface based lookup) but only allows access to them if a login has been performed first. This would effectively make the whole $\mathrm{H} 2 \mathrm{O}$ system completely transparent.

\section{Proposed Implementation}

Since we are working within a plug-in based paradigm the most natural approach is to encapsulate all the registration logic into a separate pluglet and deploy it within a $\mathrm{H} 2 \mathrm{O}$ kernel. The goal is to have the pluglet, on deployment or when triggered, detect which kernel it finds itself in and register it with a Jini LUS. The same goes for registering pluglets. In this way any $\mathrm{H} 2 \mathrm{O}$ kernel can easily be made 'Jini-aware' by simply deploying a standard registration pluglet. Regarding the proxy architecture, we opt for a configurable approach instead of forcing one particular method on the user. Depending on the method invocation handler used to generate the proxy either the "Empty Box" or "Login Enabled Smart Proxy" architecture is used. Thus it is the invocation handler object that ultimately defines the behaviour of the proxy. Besides the two implementations described above users are free to define their own invocation handler in order to customise the way method invocations are handled.

The functioning of the registration pluglet is as follows (see figure 1):

1. At deployment time, start the discovery process, register a proxy to the kernel in every discovered LUS and listen for pluglet deployment events.

- To register the kernel the pluglet first looks to see if there is a monitor pluglet available. If so, it is incorporated into the resulting proxy.

2. As soon as a deployment event arrives, obtain all relevant information from the newly deployed pluglet.

3. Use this information to generate a proxy, supporting all functional interfaces, using the specified invocation handler.

4. Register the object with the relevant Jini LUSs, and add the lease to the set of managed leases. 


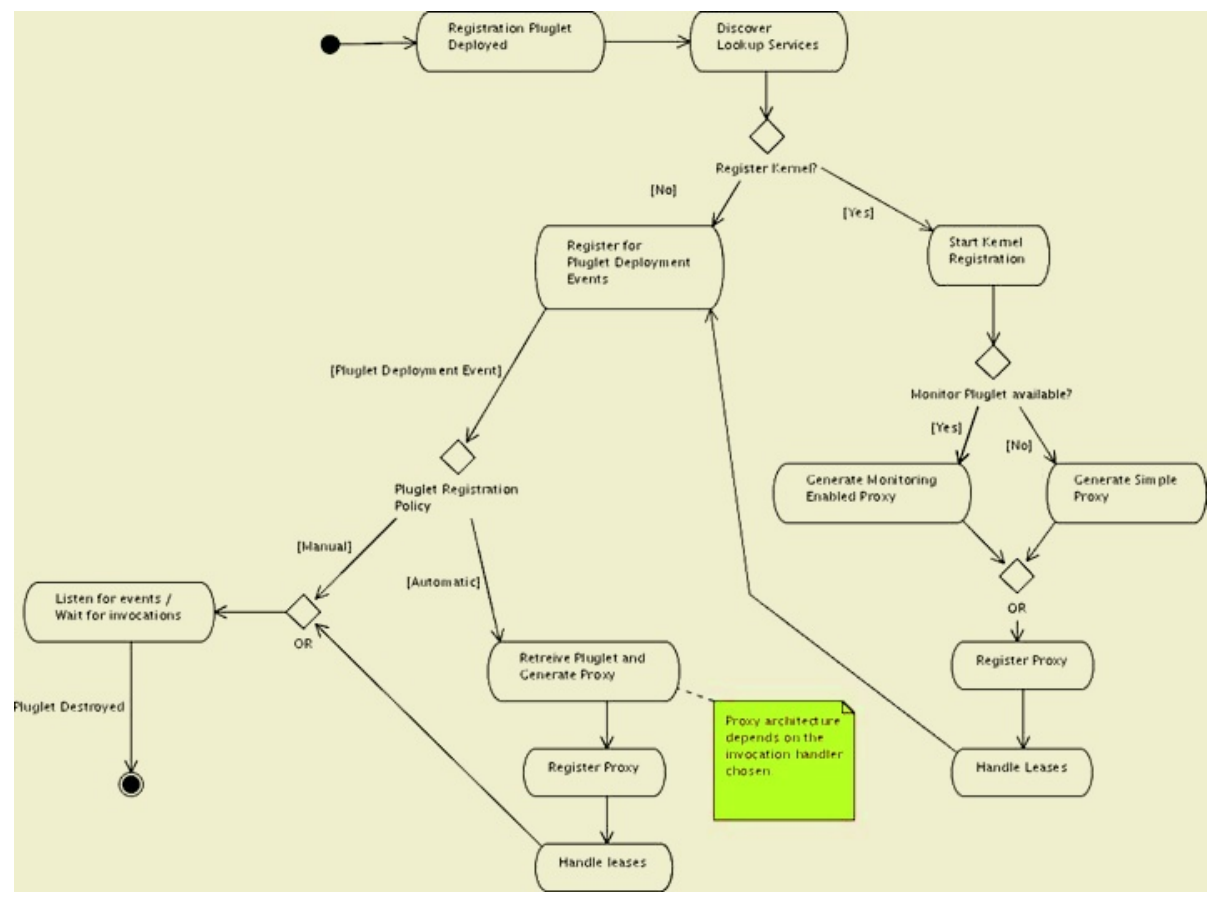

Fig. 1. Pluglet Activity Diagram

Note that if the registration pluglet receives a pluglet destroyed event, it will remove the corresponding lease form its lease map again.

In addition to the above an AutoPopulateNewLus switch further makes sure that newly discovered LUSs are automatically populated with $\mathrm{H} 2 \mathrm{O}$ resources.

Source code and documentation of the pluglet is freely available at: http://h2j.jini.org.

The pluglet described here is currently in use at the UA to maintain information about available kernels and pluglets. This information is then used by grid application developers and users to find available resources. For example, the registration pluglet is currently being used within the context of an application that simulates the quantum physical three cluster problem.

\section{$7 \quad$ Summary and Future Work}

In this paper we have demonstrated how we can use Jini to extend the H2O Metacomputing Framework with discovery and registration support. In this way $\mathrm{H} 2 \mathrm{O}$ becomes usable in a real world settings since clients no longer need a priori knowledge of service URLs. An implementation of a Jini registration pluglet was described together with the relevant design decisions. 
Future work in this area will concentrate on adding features to the registration pluglet, developing a pluglet that encapsulates core Jini services (LUS, activation daemon, event mailbox, ...) and on Jini-based $\mathrm{H} 2 \mathrm{O}$ resource management in general. An interesting avenue that may be pursued is the attachment of GUIs to each registered proxy to enable remote administration and enhance the client-side experience. This would be possible through the Jini ServiceUI infrastructure which allows one to add complete user interfaces to Jini services without any prior client-side installation.

\section{References}

1. Foster, I., Kesselman, C., Tuecke, S.: The anatomy of the grid: Enabling scalable virtual organizations. Lecture Notes in Computer Science 2150 (2001)

2. Sunderam, V., Kurzyniec, D.: Lightweight self-organizing frameworks for metacomputing. In: Proc. of 11 th IEEE International Symposium on High Performance Distributed Computing HPDC-11 20002 (HPDC'02), Edinburgh, Scotland. (2002) 119-122

3. Edwards, K.W.: Core Jini. 2 edn. Prentice Hall (2001)

4. Homburg, P., van Steen, M., Tanenbaum, A.: An architecture for a wide area distributed system. In: Proc. of 7th ACM SIGOPS European Workshop, Connemara, Ireland. (1996) 75-82

5. Schmidt, D.C., Levine, D.L., Mungee, S.: The design of the TAO real-time object request broker. Computer Communications 21 (1998) 294-324

6. Deutsch, P.: The Eight Fallacies of Distributed Computing (2004) http://java.sun.com/people/jag/Fallacies.html.

7. Hawthorne, C.: Application of Jini technology to a Swarm of Autonomous Vehicles (2003) Presentation at 7th Jini Community Meeting http://www.jini.org/meetings/seventh/J7abstracts.html\#swarm.

8. Juhasz, Z.: JGrid: Jini as a Grid technology. IEEE TFCC Newsletter (2003)

9. Juhasz, Z., Andics, A., Pota, S.: JM: A Jini Framework for Global Computing. In: Proc. of 2nd International Workshop on Global and Peer-to-Peer Computing on Large Scale Distributed Systems at IEEE International Symposium on Cluster Computing and the Grid (CCGrid'2002), Berlin, Germany. (2002) 395-400

10. Furmento, N., Lee, W., Mayer, A., Newhouse, S., Darlington, J.: ICENI: An Open Grid Service Architecture Implemented with Jini. In: Proc. of SuperComputing 2002, Baltimore, USA. (2002)

11. Y.Huang: JISGA: A Jini-based Service-oriented Grid Architecture. The International Journal of High Performance Computing Applications 17 (2003) 317-327 ISSN 1094-3420.

12. Ye, R., Hsu, W., Liu, Z., Vee, V.: Jini-enabled high performance computing. In: Proc. of 15th International Conference on Information Networking (ICOIN'01), Beppu City, Japan. (2001) $145-152$

13. Asberry, D.: The judy project, seamless integration of Jini with Webservices (2004) http://judy.jini.org.

14. Reedy, D.: Project rio: A dynamic adaptive network architecture. Technical report, Sun Microsystems (2004)

15. Creswell, D.: Jini Technology, Setting the Record Straight (2004) http://www.dancres.org/jini_setting_straight.html. 